\title{
OFTALMOPLEJÍA DOLOROSA \\ (PSEUDOTUMOR Y SÍNDROME DE TOLOSA-HUNT)
}

\author{
PAINFUL OPHTHALMOPLEGIA \\ (PSEUDOTUMOR OF THE ORBIT AND TOLOSA-HUNT \\ SYNDROME)
}

\begin{abstract}
MORA-DE-OÑATE $\mathrm{J}^{1}$, PASCUAL-PÉREZ-ALFARO R ${ }^{1}$, IZQUIERDO-VÁZQUEZ $\mathrm{C}^{1}$, GONZÁLEZ-RUIZ M ${ }^{1}$, AGUIRREBEÑA-OLMOS A ${ }^{1}$, DÍEZ-VILLALBA R ${ }^{2}$
\end{abstract}

\section{RESUMEN}

Introducción: El Síndrome de Tolosa-Hunt (STH) y el Pseudotumor Inflamatorio Orbitario (PTO) presentan tres características clínicas comunes: dolor periorbitario unilateral, parálisis de pares craneales y rápida respuesta a corticoides.

Caso clínico: Mujer de 48 años con paresia del VI par derecho, añadiéndose posteriormente paresia del III par derecho. Se plantea diagnóstico diferencial entre STH y PTO, resolviéndose en base a pruebas complementarias.

Discusión: Aparte de las pruebas neuroradiológicas, la presentación clínica e histopatológica similar hace que sean dos enfermedades difíciles de diferenciar, discutiéndose los aspectos comunes de ambas enfermedades.

Palabras clave: Pseudotumor orbitario, síndrome de Tolosa-Hunt.

\begin{abstract}
Introduction: The Tolosa-Hunt Syndrome (THS) and the Pseudotumor of the Orbit (PTO) each have three common clinical hallmarks: unilateral periorbital pain, cranial nerve palsies and a fast response to corticosteroid therapy.

Case Report: 48-year-old female with a right VI nerve paralysis, later develops a right III nerve paralysis. It is important to be able to differentiate between THS and PTO, and this is done on the basis of the complementary tests.
\end{abstract}

Discussion: Apart from neuroradiologic findings, the clinical presentation and histopathology of these two conditions are very similar, making it difficult to distinguish between them. Aspects common to both pathological processes are discussed (Arch Soc Esp Oftalmol 2007; 82: 509-512).

Key words: Pseudotumor of the orbit, Tolosa-Hunt syndrome.

\footnotetext{
Recibido: 31/5/06. Aceptado: 19/6/07.

Departamento de Oftalmología, Hospital Universitario de Getafe, Madrid, España.

1 Licenciado en Medicina.

2 Doctor en Medicina.

Correspondencia:

Javier Mora de Oñate

$\mathrm{C} /$. Marqués de Mondéjar, 20 - 3 . $^{\circ} \mathrm{B}$

28020 Madrid

España

E-mail: javimora79@hotmail.com
} 


\section{INTRODUCCIÓN}

El síndrome de oftalmoplejía dolorosa consiste en un cuadro de dolor periorbitario o hemicraneal, combinado con parálisis ipsilateral del nervio oculomotor común, y pérdida de sensibilidad en la distribución de la rama oftálmica y/o maxilar del nervio trigémino (1).

La etiología de Oftalmoplejía dolorosa se reseña en la tabla I (2).

Dentro de este síndrome hay dos causas difíciles de diferenciar: el Pseudotumor Orbitario (PTO) y el Síndrome de Tolosa-Hunt (STH). Ambas patologías son procesos inflamatorios crónicos granulomatosos de origen desconocido que presentan muchas características clínicas y diagnósticas en común (3).

A pesar de que se ha avanzado en el conocimiento de estas enfermedades, el PTO sigue siendo la tercera patología orbitaria después de la oftalmopatía tiroidea y enfermedades linfoproliferativas (4). Aunque, sin embargo, el término pseudotumor orbitario últimamente viene siendo sustituido por el de «inflamación orbitaria no específica o idiopática» ya que cada vez es menor el número de casos en el que no se encuentra etiología.

Para el diagnóstico diferencial de ambos síndromes es esencial hacer una correcta historia clínica así como realizar las pruebas complementarias pertinentes.

Los criterios diagnósticos del STH se reseñan en la tabla II (2).

\section{Tabla II. Criterios diagnósticos del STH}

A Uno o más episodios de dolor orbitario unilateral que persiste durante semanas sin tratamiento.

B Parálisis de uno o más de los nervios oculomotores (III, IV o VI) y/o demostración de granuloma en RM cerebral o biopsia.

C La oftalmoparesia coincide con el comienzo del dolor o le sigue en menos de dos semanas.

D El dolor y la parálisis se resuelven en 72 horas cuando se instaura un correcto tratamiento corticoideo.

E Exclusión de otras causas mediante investigaciones adecuadas.

Es importante descartar la presencia de un tumor maligno mediante Resonancia Magnética Cerebral (RMC) y/o Tomografía Computerizada y un seguimiento exhaustivo al menos durante dos años (4).

En ambos casos los corticoides e inmunosupresores suelen ser el tratamiento de elección, ya que producen la desaparición de los síntomas a los pocos días de su instauración, siendo parte de los criterios diagnósticos en el STH (4).

\section{CASO CLÍNICO}

Mujer de 48 años con antecedentes de migraña con y sin aura oftálmica, fumadora, que acude a urgencias por presentar visión borrosa en OD y diplopia binocular de dos días de evolución. En la exploración oftalmológica se evidenció una limita-

\section{Tabla I. Causas de Oftalmoplejía dolorosa}

\section{Traumatismos}

Fractura de la base del cráneo
Migraña Oftalmopléjica

Infecciosas
Sinusitis
Mucormicosis
Tuberculosis
Sífilis
Enfermedad de Lyme

Trombosis del seno cavernoso

Neuropatía diabética

\section{Tumorales}

Tumores primarios

Meningiomas

Craneofaringiomas

Neurinomas

Adenomas hipofisarios

Tumores metastáticos

Nasofaringe

Linfoma

Mieloma múltiple
Inflamatorias Idiopáticas

Oftalmopatía tiroidea

Sarcoidosis

Vasculitis

Lupus Eritematoso Sistémico

Arteritis de células gigantes

Granulomatosis de Wegener

Síndrome de Tolosa-Hunt

Pseudotumor Orbitario 
ción a la abducción del ojo derecho y se diagnosticó de parálisis del VI par derecho. El resto de la exploración normal. Se realizaron de forma ambulatoria los siguientes estudios complementarios:
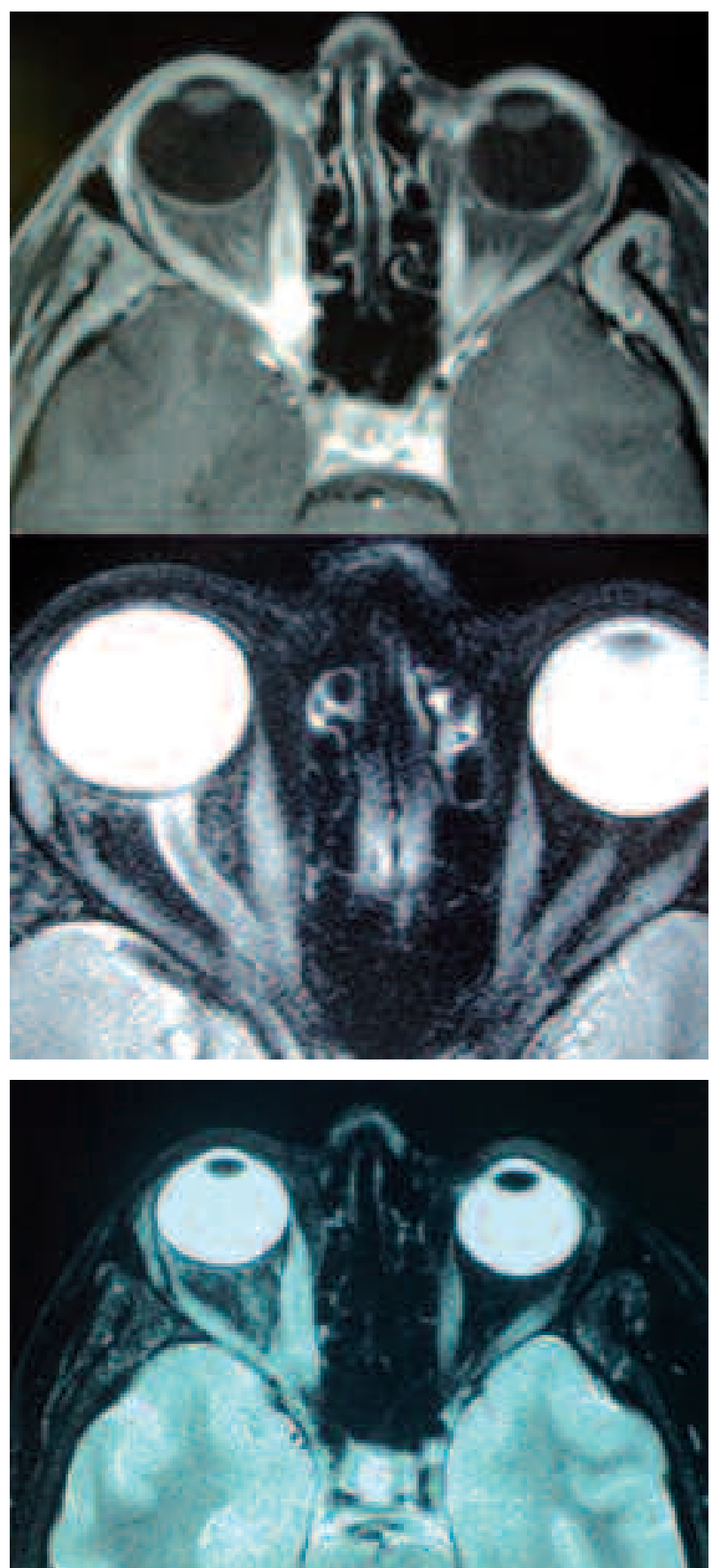

Figs. 1 y 2: Corte axial de RMN de ambas orbitas en la que se evidencia un engrosamiento difuso del músculo recto medial derecho sin afectación del recto lateral derecho. hemograma y bioquímica normal, hormonas tiroideas, anticuerpos microsomales, anticuerpos antitiroglobulinas, serología lúes, factor reumatoide C3C4, coagulación básica y VSG normales. Radiografía de tórax sin hallazgos. TC craneal sin alteraciones. Dúplex de troncos supraaórticos normal. Mientras se realizan estos estudios, vuelve a urgencias por presentar dolor periorbitario y malar derecho, con empeoramiento nocturno, que no cede con AINEs. En la exploración se objetiva una mayor limitación de la abducción OD sin parestesias faciales, con resto de la exploración normal. Días después se pone de manifiesto una limitación de la supraducción y la infraducción, con AV con corrección OD 0,6 con agujero estenopeico 0,8; OI 1. La exploración de fondo de ojo permanece normal. Se le realiza en ese momento una campimetría, con defectos centrales de mayor intensidad en lado temporal del OD, siendo la del OI normal.

Se solicita una RMN craneal con estudio en secuencias potenciadas T1-T2, densidad protónica T1 con gadolinio de ambas órbitas, demostrándose la presencia de unos músculos oculares aumentados de tamaño, sobretodo a expensas del recto medial y del recto inferior derecho (figs. 1 y 2). No hay evidencias de alteraciones del seno cavernoso (fig. 3).

Debido a estos hallazgos en la RMN además de la posibilidad de una parálisis del VI par craneal y otras posibles afectaciones oculomotoras que se consideraron al principio, se añade la restricción de la motilidad ocular por el engrosamiento e inflamación de la miositis como causa de la limitación de la abducción, supraducción e infraducción.

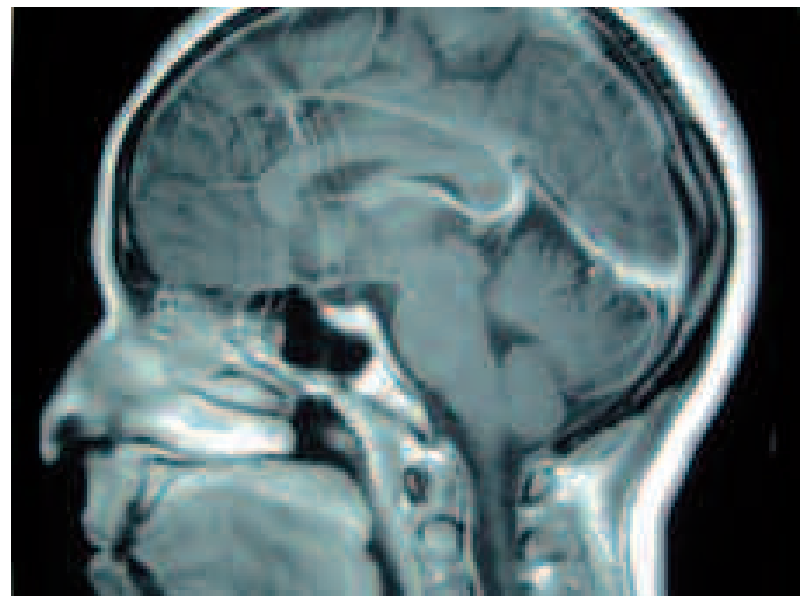

Fig. 3: Corte sagital de RMN craneal. Se demuestra ausencia de alteraciones en el seno cavernoso. 


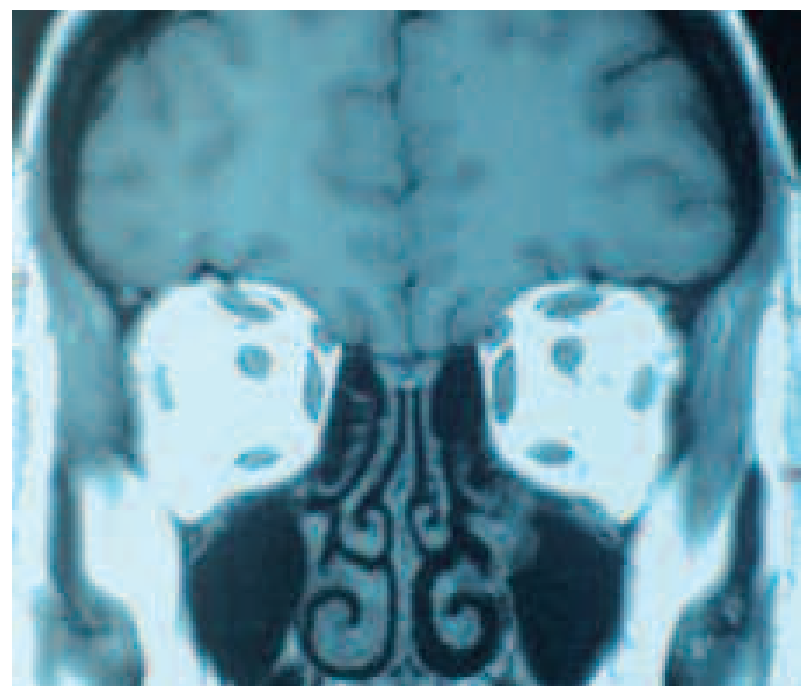

Fig. 4: Corte coronal de RMN. Remisión del engrosamiento del músculo recto medial derecho a los dos meses tras iniciar el tratamiento con corticoides.

Se le realiza punción lumbar. El LCR presentaba presión de apertura, celularidad, glucosa, proteínas y hematíes en niveles de normalidad.

Se comienza tratamiento con Metilprednisolona intravenosa en dosis de $250 \mathrm{mg} /$ día durante 3 días con importante mejoría del dolor y de la paresia sobretodo de los músculos recto superior, recto interno, oblicuo menor y recto inferior. También hubo mejoría del músculo recto externo. Se mantiene tratamiento con Prednisona $60 \mathrm{mg} /$ día durante 10 días continuándose con una pauta descendente durante un mes. Al final del tratamiento se consigue una remisión completa del cuadro con desaparición de los síntomas y de los hallazgos radiológicos (fig. 4).

\section{DISCUSIÓN}

El caso clínico presentado corresponde a una afectación inflamatoria orbitaria (pseudotumor orbitario), sin datos en el LCR o RM Craneal o en perfil evolutivo que sugieran afectación de seno cavernoso por enfermedad inflamatoria asociada (síndrome de Tolosa-Hunt).
La dificultad de clasificar la oftalmoplejía dolorosa nos debe hacer ser muy meticulosos a la hora de interrogar y explorar al paciente así como tener que recurrir a pruebas complementarias específicas para poder llegar a un diagnóstico de certeza.

Las pruebas de imagen son importantes ya que al compartir ambas patologías muchas características clínicas, son ellas las que nos permiten diferenciar la mayor parte de las manifestaciones del pseudotumor orbitario como miositis (nuestro caso), dacrioadenitis, periescleritis, perineuritis, o infiltración de grasa infraorbitaria, dejando al Síndrome de Tolosa-Hunt como un diagnóstico de exclusión.

Hay casos, sin embargo, aunque en un bajo porcentaje, que se presentan con manifestaciones clínicas poco características en las que los hallazgos radiológicos son inespecíficos. En estos casos es importante realizar un exhaustivo diagnóstico diferencial y descartar otras entidades, como por ejemplo oftalmopatía tiroidea y enfermedades linfoproliferativas. En el pseudotumor los tendones se engrosan junto con los vientres musculares adquiriendo una configuración tubular. Esto contrasta con la oftalmopatía tiroidea en la que los músculos adquieren una configuración más alargada con tendones normales. Un engrosamiento marcado de un solo músculo podría ser confundido con un tumor (5).

\section{BIBLIOGRAFÍA}

1. Kline LB, Hoyt WF. The Tolosa-Hunt syndrome. J Neurol Neurosurg Psychiatry 2001; 71: 577-582.

2. Jiménez-Caballero PE, Florensa J, Marsal-Alonso C, Álvarez-Tejerina A. Síndrome de Tolosa-Hunt de repetición con neuroimagen normal. Descripción de tres casos. Rev Neurol 2005; 41: 30-33.

3. Wasmeier C, Pfadenhauer K, Rosler A. Idiopathic inflammatory pseudotumor of the orbit and Tolosa-Hunt Syndrome-are they the same disease? J Neurol 2002; 249: 1237-1241.

4. Rubin PA, Foster CS. Etiology and management of idiopathic orbital inflammation. Am J Ophthalmol 2004; 138: 1041-1043.

5. Weber AL, Romo LV, Sabates NR. Pseudotumor of the orbit, clinical, pathologic, and radiologic evaluation. Radiol Clin North Am 1999; 37: 151-168. 